Research in Astron. Astrophys. Vol.0 (20xx) No.0, 000-000

http://www.raa-journal.org http://www.iop.org/journals/raa

$\boldsymbol{R}$ esearch in

(LTEX: tgss.tex; printed on February 4, 2019; 1:38)

Astronomy and

Astrophysics

1

Key words: galaxies: high-redshift, galaxies: active galactic nuclei

\title{
Radio spectral index from NVSS and TGSS
}

\author{
Prabhakar Tiwari \\ National Astronomical Observatories, Chinese Academy of Sciences \\ Beijing, 100012, China \\ ptiwari@nao.cas.cn
}

\begin{abstract}
I extract the radio spectral index, $\alpha$, from 541,195 common sources observed in the $150 \mathrm{MHz}$ TIFR GMRT Sky Survey (TGSS) and the $1.4 \mathrm{GHz}$ NRAO VLA Sky Survey (NVSS). This large common source catalogue covers about $80 \%$ of the sky. The flux density limits in these surveys are such that the observed galaxies are presumably hosts of active galactic nuclei (AGNs). I confirm the steepening of $\alpha$ with increasing flux density for this large sample and provide a parametric fit between $\alpha$ and flux density. Next, I divide the data into a low flux (LF) and a high flux (HF) density sample of roughly equal number of galaxies. The LF sample contains all galaxies below 100 mJy TGSS and 20 mJy NVSS flux density and the HF sample is all galaxies above $100 \mathrm{mJy}$ TGSS and 20 mJy NVSS. I observe an increase in $\alpha$ with source size (TGSS measured), saturating for large sizes to $0.89 \pm 0.22$ and $0.76 \pm 0.21$ for the LF and HF sources, respectively. I discuss the observed results and possible physical mechanisms to explain observed $\alpha$ dependence with source size for LF and HF samples.
\end{abstract}

\section{INTRODUCTION}

The radio emission from a distant galaxy consists of thermal and synchrotron contributions. The thermal emission is bremsstrahlung radiation from HII regions while the non-thermal synchrotron emission is generated by supernova remnants (SNRs), diffuse cosmic ray electrons (CREs) spread over the disk and halo (Biermann 1976; Condon 1992), relativistic plasma in jets and jet fed lobes (De Young 1976; Blandford \& Königl 1979). The bright radio galaxies, relevant to flux density limits in this work, are hosts of AGNs and their radio emission is predominantly synchrotron radiation from jets of relativistic plasma and jet fed lobes ${ }^{1}$. These emission components are characterized by different spectral indices and, therefore, the total spectral index depends on their relative contributions. The thermal emission is almost flat $\left(\propto \nu^{-\alpha}\right)$ with spectral index $\alpha_{\mathrm{th}} \approx 0.1$ (Condon 1992), whereas, the radio jets exhibit a spectral index $\alpha_{\text {jet }}=0.5-0.7$ (Bridle \& Perley 1984; Laing \& Bridle 2013) and the radio emission from lobes can be flat to very steep depending on energy injection and losses (Kellermann 1966).

I compile a common source catalogue from TIFR GMRT Sky Survey first alternative data release (TGSS ADR1) (Intema et al. 2017) and NRAO VLA Sky Survey (NVSS)(Condon et al. 1998) catalogues, and use it to explore radio spectral index, size, and flux density dependence. I identify a total of 541,195 sources which are common to both TGSS ADR1 and NVSS. The large sample allows us to separate spectral index values in to differential source size bins and I obtain statistically significant dependence of spectral index on flux density and size. Assuming that the NVSS source population peaks

\footnotetext{
1 and the inverse-Compton scattering of the radio synchrotron emission within the lobes
} 
between redshift $z \approx 0.5-1$ (Wilman et al. 2008; Nusser \& Tiwari 2015; Tiwari \& Nusser 2016), all results in this work statistically represents the radio source population at redshift $z \approx 0.8$.

The outline of the paper is as following. I discuss the NVSS and TGSS ADR1 data and describe the cross-matching procedure in Section 2. In Section 3 I discuss the basic mechanisms of radio emission and their spectral index. I describe the observed spectral index in Section 4. In Section 5 I present all results. I conclude with discussion in Section 6.

\section{DATA}

\subsection{TGSS ADR1}

The TGSS ADR1 catalogue is based on the Giant Metrewave Radio Telescope (GMRT), performing all-sky radio continuum survey at $150 \mathrm{MHz}$ (Swarup 1991). The catalogue is surveyed between 2010 and 2012 and has been published recently by Intema et al. (2017). The catalogue covers $90 \%$ of the full sky at $150 \mathrm{MHz}$ with median RMS brightness fluctuations $3.5 \mathrm{mJy} / \mathrm{beam}$ with approximate resolution $25^{\prime \prime} \times 25^{\prime \prime}$ north of $19^{\circ} \mathrm{DEC}$ and $25^{\prime \prime} \times 25^{\prime \prime} / \cos \left(\mathrm{DEC}-19^{\circ}\right)$ south of $19^{\circ}$. The best resolution observed with GMRT at $150 \mathrm{MHz}$ is $20^{\prime \prime}(\mathrm{Lal} 2013)$ and between declination $-20^{\circ}$ to $+60^{\circ}$ TGSS ADR1 sources are best resolved with resolution close to $20^{\prime \prime}$ (see figure 6 in Intema et al. (2017)). The catalogue contains a total 623,604 sources $\left(489,570\right.$ sources above flux density $\left.{ }^{2} 50 \mathrm{mJy}\right)$ observed at $6 \sigma$ peak-to-noise threshold. The accuracy of the source centroid position is better than $2^{\prime \prime}(\mathrm{RMS})$. The catalogue is almost complete above $100 \mathrm{mJy}$ (Intema et al. 2017) and 70\% complete above $50 \mathrm{mJy}$ at $150 \mathrm{MHz}$.

\subsection{NVSS}

The NVSS catalogue contains $\sim 1.8$ million sources with flux densities $S_{1.4 \mathrm{GHz}}>2.5 \mathrm{mJy}$ at 1.4 $\mathrm{GHz}$ (Condon et al. 1998). The full width at half maximum angular resolution is $45^{\prime \prime}$ and nearly all observations are performed at uniform sensitivity. The catalogue covers about $82 \%$ of the sky and has a 100\% overlap with TGSS ADR1. The RMS uncertainty in angular position is up to $7^{\prime \prime}$, substantially larger than TGSS. The catalogue is complete above $3.5 \mathrm{mJy}$ at $1.4 \mathrm{GHz}$.

\subsection{TGSS-NVSS common source catalogue}

Given the TGSS angular position of each of the 623,604 TGSS ADR1 sources, I identify NVSS sources within an angular distance less than $30^{\prime \prime}$. In total I find 553,301 TGSS ADR1 sources having at least one NVSS source within $30^{\prime \prime}$. Given the positional uncertainties of NVSS (Section 2.2) and TGSS ADR1 (Section 2.1) and with $30^{\prime \prime}$ criteria we have a less than 4 sigma chance to miss a source. Therefore due to positional uncertainty, we expect only to miss a few tens of sources while identifying NVSS match for 0.6 million TGSS ADR1 sources. Furthermore, given the NVSS source number density, Section 2.2, there is a finite probability, $\approx 0.013$, of having a NVSS source in $30^{\prime \prime}$ radius and we expect to misidentify around 8000 sources due to this probability. Due to all these and other possible observational systematics and uncertainties (Condon et al. 1998; Intema et al. 2017) I find 12,106 sources, out of 553,301 cross-matched, these are either showing more than one NVSS association with search criteria of $30^{\prime \prime}$ or more than one TGSS sources showing association with same NVSS object. I drop these 12,106 sources to avoid confusion and artificial scatter in the spectral index. I end up with a total 541,195 sources, identified in both TGSS ADR1 and NVSS. The common source counts for different TGSS flux density cuts are listed in Table 1. The spatial distribution of these common sources is shown in Fig. 1.

\footnotetext{
2 "flux density" refers to total flux density throughout the paper.
} 


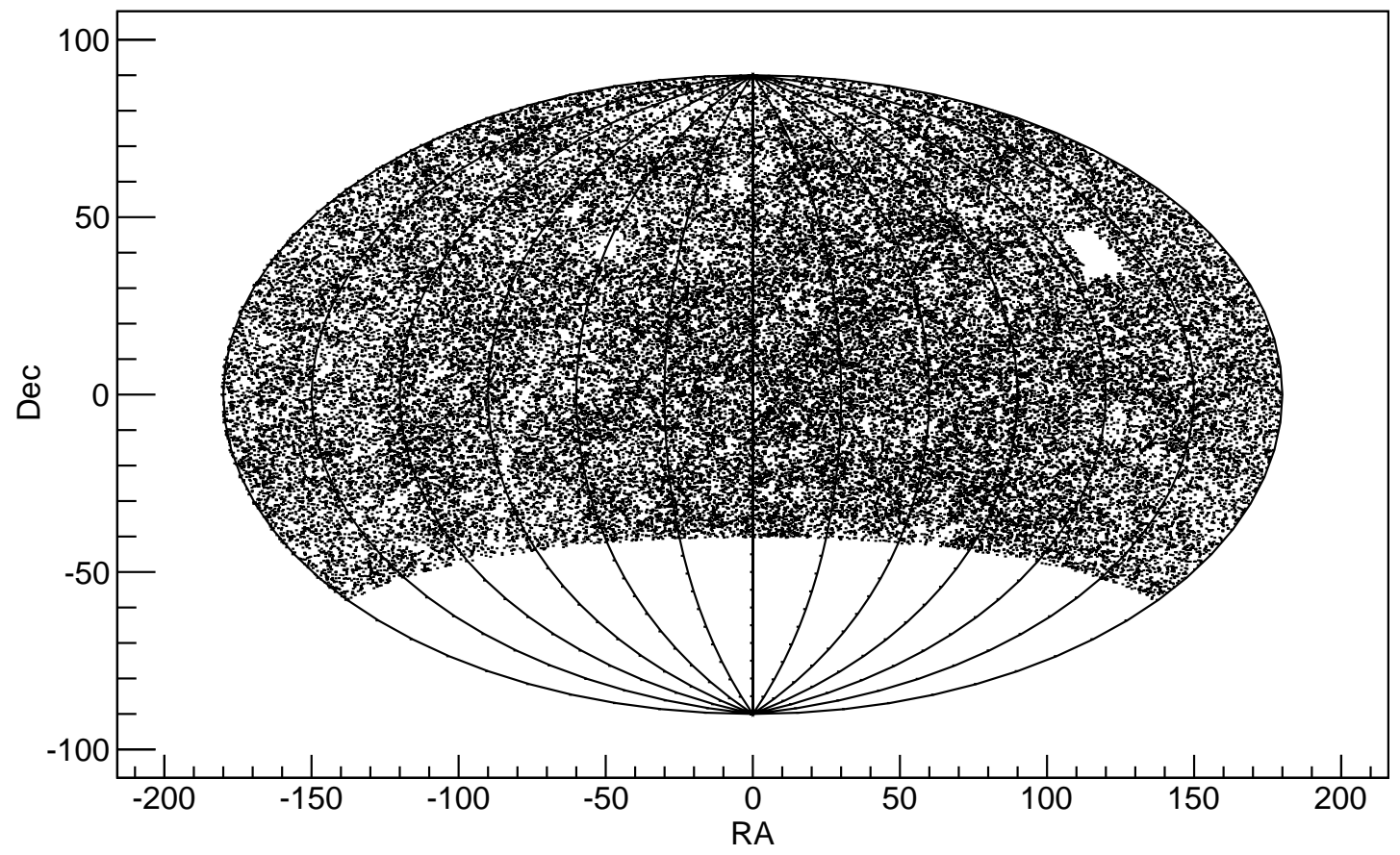

Figure 1: The angular distribution of NVSS-TGSS common sources. Only 10\% randomly selected sources are plotted.

Table 1: TGSS-NVSS common sources with different TGSS flux density cuts.

\begin{tabular}{cc}
\hline $\begin{array}{c}\text { STGSS } \\
\text { (mJy) }\end{array}$ & number of sources \\
\hline no cut & 541,195 \\
$>50$ & 424,639 \\
$>100$ & 266,885 \\
$>150$ & 190,830 \\
$\geq 200$ & 147,171 \\
\hline
\end{tabular}

\section{RADIO EMISSIONS IN AGNS}

\subsection{Thermal radio emission from HII regions}

The flux density of radio thermal emission dependence on frequency is expressed as (Condon 1992),

$$
S_{T} \propto \nu^{-0.1} .
$$

At high frequencies $(\sim \mathrm{GHz})$ where the electron deflection opacity is small, the ratio of the non-thermal emission approximate thermal radio emission $S_{T}$ with respect to non-thermal radio emission $S_{\mathrm{NT}}$ is (Condon \& Yin 1990),

$$
S_{\mathrm{NT}} \sim 10\left(\frac{\nu}{\mathrm{GHz}}\right)^{0.1-\alpha} S_{T},
$$

where $\alpha \sim 0.8$ is the approximate non-thermal spectral index (Intema et al. 2011; Williams et al. 2013, 2016; Hardcastle et al. 2016; Mahony et al. 2016b). This implies that the non-thermal emission is dominant with a thermal contribution of $\lesssim 10 \%$ at $1.4 \mathrm{GHz}$ and $\lesssim 3 \%$ at $150 \mathrm{MHz}$. 


\subsection{Non-thermal radio emission}

The radio galaxies are broadly categorized in Fanaroff \& Riley (1974) type I (FR I) and II (FR II), and the large-scale structure of a radio galaxy is roughly consist of three components: jets, lobes and hotspots (Harwood et al. 2013, 2015). In FR I galaxies, the jets are relatively less collimated and produce radio emission trough deceleration (Laing \& Bridle 2013, 2014), whereas the FR II jets are often non-emitting and the radio emission largely produced in lobes and hotspots (Harwood et al. 2013, 2015).

\subsubsection{Radio emission from jets}

The relativistic jet transports the material from central AGN to extremities of the source and terminates as shock forming a hotspot i.e. a compact region of synchrotron emission. At the point of acceleration, the electrons number distribution is expressed as $N(E) \propto E^{-\gamma}$, where $\gamma \approx 2$ for FR I (Blandford \& Königl 1979) and $\gamma>2$ for FR II (Harwood et al. 2016, 2017b). In FR I galaxies, for which the emission form jets is significant, the mean spectral index of radio emission from jets decreases with distance from the nucleus and $\alpha_{\text {jet }} \approx 0.5-0.7$ (Laing \& Bridle 2013).

\subsubsection{Radio emission from Jet fed lobes}

The radio emission from extended radio-loud galaxies is dominant by radio lobes which are fed by collimated narrow jets. The radio emission from these lobes depends on electron injection and energy losses. For a continuous supply of electrons, the number distribution obtained by considering losses to synchrotron is $N(E) \propto E^{-1-\gamma}$ (Kellermann 1966), corresponding to $\alpha=\gamma / 2$. If the energy injection is intermittent, then $\alpha$ depends on the frequency $\nu$ and the time interval between the bursts. In this case, the first-order Fermi acceleration assuming strong shocks (Bell 1978; Blandford \& Ostriker 1978; Kirk et al. 2000; Lemoine \& Pelletier 2003) gives relatively flat spectrum $\left(\alpha_{\text {lobes }} \geq 0.5\right)$ at low frequencies and very steep $\left(\alpha_{\text {lobes }}=1.33\right)$ at higher frequencies (Kellermann 1966).

\subsubsection{Radio emission from core}

The spectral characteristics of "compact" (i.e., unresolved at $\sim 1$ " resolution) radio sources is very different from "extended" sources. The radio emission from the core is significant for compact radio sources (Antonucci 1993; Urry \& Padovani 1995) and assuming the power-law distribution of electron energies, the radio spectra of compact sources are usually flat with $\alpha_{\text {core }} \leq 0.5$ (Peterson 1997). In particular for compact blazars the radio emission from the core dominates and the radio spectral index is $\approx 0.0$ (Fan et al. 2010).

\section{OBSERVED SPECTRAL INDEX}

I derive the spectral index, $\alpha$, from the measured radio emission at frequencies $\nu_{\mathrm{TGSS}}=150 \mathrm{MHz}$ and $\nu_{\mathrm{NVSS}}=1.4 \mathrm{GHz}$ for objects in the TGSS-NVSS common source catalogue. For each source I compute the observed spectral index as,

$$
\alpha_{\mathrm{obs}}=\frac{\ln \left(\mathrm{S}_{\mathrm{TGSS}} / \mathrm{S}_{\mathrm{NVSS}}\right)}{\ln \left(\nu_{\mathrm{NVSS}} / \nu_{\mathrm{TGSS}}\right)},
$$

where $\mathrm{S}_{\text {TGSS }}$ and $\mathrm{S}_{\text {TGSS }}$ are, respectively, the flux densities measured by the TGSS and NVSS. As we have seen in Section 3.1, thermal emission is negligible and this $\alpha_{\mathrm{obs}}$ is mainly fixed by the synchrotron component.

I divide the catalogue of common source in to two samples. The first low flux (LF) density sample contains sources with TGSS and NVSS flux densities below $100 \mathrm{mJy}$ and $20 \mathrm{mJy}$, respectively ${ }^{3}$. The second high flux $(\mathrm{HF})$ density sample includes sources above and equal $\mathrm{S}_{\mathrm{TGSS}}=100 \mathrm{mJy}$ and $\mathrm{S}_{\mathrm{NVSS}}=$

\footnotetext{
${ }^{3}$ Note that $\mathrm{S}_{\mathrm{TGSS}}=100 \mathrm{mJy}$ corresponds to $\mathrm{S}_{\mathrm{NVSS}} \approx 20 \mathrm{mJy}$, assuming spectral index $\approx 0.73$.
} 
20 mJy. These flux density cuts are imposed so that both samples LF and HF contain roughly $50 \%$ of sources from common source catalogue. We notice from Wilman et al. (2008) (see figure 4 therein) simulation that the LF sample is expected to consists of mostly FR I galaxies whereas HF sample supposed to contain almost equal number of FR I and FR II galaxies. Furthermore, note that the TGSS ADR1 catalogue is approximately complete above $100 \mathrm{mJy}$ and so the HF sample represents the full radio source population (Intema et al. 2017). The LF sample is increasingly incomplete for lower flux densities. Nonetheless, LF sample represents the sources with low flux density.

The non-thermal radio emission consists mainly of synchrotron emission from jets and lobes. The spectral index from such systems is not accurately known. For jets the spectral index $\alpha_{\text {jet }}$ ranges from 0.5 to 0.7 (Laing \& Bridle 2013) and for lobes the nor-thermal emission may be anywhere between 0.5 to 1.33 (Bell 1978; Blandford \& Ostriker 1978; Kirk et al. 2000; Lemoine \& Pelletier 2003; Kellermann 1966). In general we can not isolate the radio emission from jets and lobes, however, the relative contribution from lobes dominates in large sources. For ultra large sources and for continuous energy injection into the lobes, $\alpha_{\text {lobes }}$ saturates to the value $\gamma / 2$, where $\gamma$ refers to the electrons in the lobes.

\section{RESULTS}

\subsection{Radio spectral index dependence on flux density}

Fig. 2 and 3 show the distribution of $\alpha_{\text {obs }}$ for sources with different flux density cuts and bins, respectively. Although the RMS spread is large, we see a clear trend of steepening of the spectral index with increasing flux density. The error in spectral index measurement due to flux density uncertainty is (Mahony et al. 2016b),

$$
\delta \alpha=\frac{1}{\ln \frac{\nu_{1}}{\nu_{2}}} \sqrt{\left(\frac{\delta S_{1}}{S_{1}}\right)^{2}+\left(\frac{\delta S_{2}}{S_{2}}\right)^{2}}
$$

where $\nu_{1,2}$ and $S_{1,2}$ referes to NVSS and TGSS frequencies and flux densities respectively. The flux density accuracy in TGSS ADR1 and NVSS is $\sim 10 \%$ (Intema et al. 2017) and $\sim 5 \%$ (Condon et al. 1998), respectively. Using equation 4 , these flux density uncertainties give $\delta \alpha \sim 0.05$. However, the RMS spread observed in Fig. 2 is about 0.24 which is much larger than that expected from flux density measurement uncertainty. Thus the RMS spread observed is largely the intrinsic distribution of source spectral indices. This is expected since the individual sources have very different radio emission properties, i.e. it may be FR I, FR II type and its radio flux may be largely from jets or lobes. To explore this further, I sort the sources in logarithmic bins of $\mathrm{S}_{\mathrm{TGSS}}$ and plot the mean spectral index $\bar{\alpha}_{\text {obs }}$ in each bin as a function of the flux density in Fig. 4. The observed dependence is fitted by linear parametric form as indicated in the figure. This confirms the earlier hints of spectral index dependence on flux density (Gopal-Krishna \& Steppe 1982; Steppe \& Gopal-Krishna 1984; Windhorst et al. 1993; Prandoni et al. 2006; Ibar et al. 2009; Ishwara-Chandra et al. 2010; Randall et al. 2012). However this trend of increasing spectral index is exclusinve to flux density bins of $\mathrm{S}_{\mathrm{TGSS}}$ i.e. to low frequency bins. The trend is almost inverted if we sort the sources in logarithmic bins of $S_{\text {NVSS }}$ and plot the mean spectral index (Fig. 5). This is expected and evident from equation $3, \frac{d \alpha_{\text {obs }}}{d \ln \mathrm{S}_{\mathrm{TGSS}}}$ and $\frac{d \alpha_{\mathrm{obs}}}{d \ln \mathrm{S}_{\mathrm{NVSS}}}$ are positve and negative, respectivelly.

The TGSS ADR1 data has high noise in the Galactic plane (Intema et al. 2017); furthermore, there are compact bright source present in the Galactic plane (White et al. 2005) with significant thermal emission. Therefore, I mask the Galactic plane to check the robustness of the results. I find that masking the Galactic plane by 5 or 10 degrees changes $\bar{\alpha}_{\text {obs }}$ in Fig. 2 by a tiny value $<0.008$. This is so tiny that the fit in Fig. 4 remains unchanged.

A small fraction of radio source population is known to exhibit peaked-spectrum or convex spectrum between the TGSS and NVSS frequencies (Callingham et al. 2017). These sources can be identified if the flux density is known in a large fractional bandwidth e.g. the GaLactic and Extragalactic All-sky Murchison Widefield Array (GLEAM) catalogue (Hurley-Walker et al. 2017). Ideally, I should mask all peaked-spectrum sources as these source do not follow equation 3. However, as we have flux 


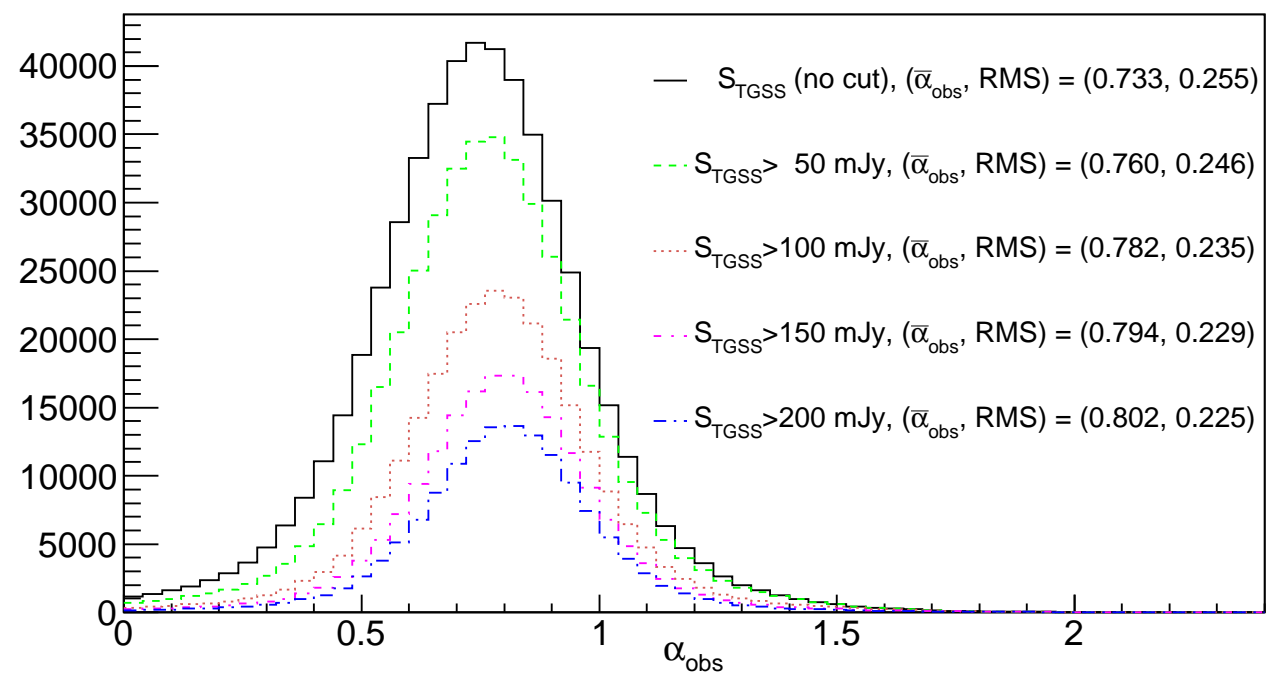

Figure 2: The NVSS-TGSS extracted radio spectral index with various TGSS flux density cuts. The RMS is the root mean square from $\alpha_{\text {obs }}$ distribution. The TGSS ADR1 is complete above $S_{\mathrm{TGSS}}>100 \mathrm{mJy}$.

densities only at two frequencies we can not identify peaked-spectrum sources. Nevertheless, I use Callingham et al. (2017) peaked-spectrum source catalogue prepared from GLEAM survey, which covers $\sim 60 \%$ of TGSS-NVSS surveys, and identify in total 1069 peaked and 83 convex spectrum sources in our common catalogue. I mask these sources and find that the mean spectral index in Fig. 2 changes by $<0.003$. The remaining $40 \%$ of TGSS-NVSS sky, not covered by GLEAM, is expected to have similar fraction of peaked-spectrum sources, i.e. around one thousand more peaked-spectrum sources, even so we do not expect any significant change in the results. Furthermore we expect a few percent of mJy radio sources to be variable on the time-scale of years (Oort \& Windhorst 1985; Fan et al. 2007). The unresolved compact sources e.g. blazars show more than $4 \sigma$ flux varibility and constitutes a small faction of total radio population. For example $0.1 \%$ of the unresolved sources from FIRST and NVSS are found to be variable Ofek \& Frail (2011). Similarly Bell et al. (2019) report that $\sim 0.15 \%$ of bright compact sources at low frequencies ( $>4 \mathrm{Jy}$ at $154 \mathrm{MHz}$ ) show significant long-term variability. In our case variable sources may constitute not more than a few percentage of total population and may show very steep or flat spectral index and contribute to the outliers of histograms in Fig. 2 and 3, however, this is not expected to change the mean spectral index significantly.

\subsection{Radio spectral index dependence on size}

The size is defined as $\sqrt{a b}$ (in arcsec) where $a$ and $b$ are, respectively, the major and minor axis of the Gaussian fit to the source as given in TGSS ADR1. As mentioned in Section 4, the observed spectral index consists of radio emission from jets and lobes. Fig. 6 shows $\alpha_{\text {obs }}$ as a function of size for LF sample. The mean $\alpha_{\text {obs }}$ is computed for sources lying in logarithmic bins of $\sqrt{a b}$. The spectral index increases rapidly with size until $\leq 26^{\prime \prime}$ but flattens at larger sizes, becomes nearly independent of size beyond $\sim 44^{\prime \prime}$ and saturates to the value $\alpha_{\text {obs }}=0.893 \pm 0.223$. 


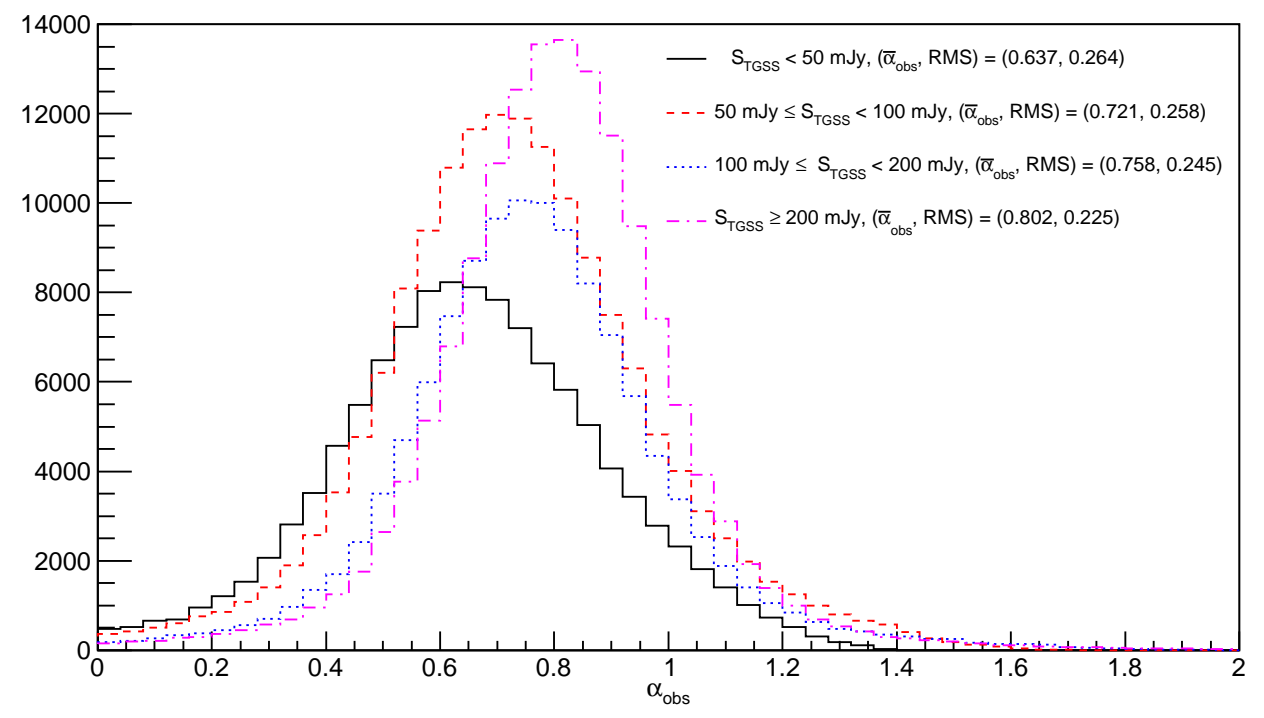

Figure 3: The NVSS-TGSS extracted radio spectral index in various TGSS flux density bins.

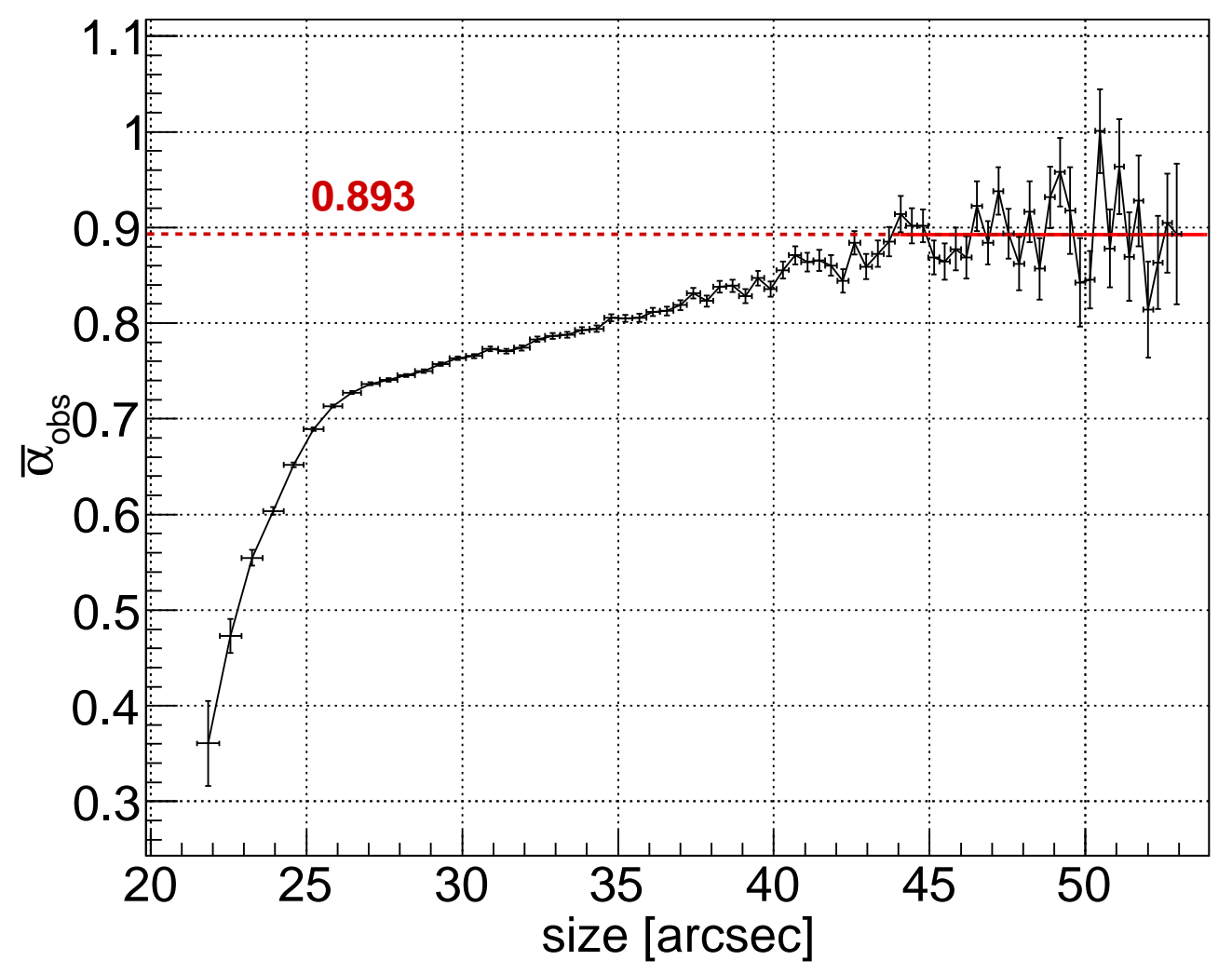

Figure 6: The mean radio spectral index, $\bar{\alpha}_{\text {obs }}$, dependence on size $(\sqrt{a b})$ for sources in the LF sample. The vertical and horizontal error-bars represent the uncertainty in $\bar{\alpha}_{\text {obs }}$ and the width of the bin. The $\bar{\alpha}_{\text {obs }}$ dependence on size saturates very clearly for the sources above 44 arcsec (roughly $340 \mathrm{kpc}$ for $z \approx 0.8$ ). The red dashed line shows the mean $\alpha_{\text {obs }}=0.893 \pm 0.223$ from sources above 44 arcsec. 


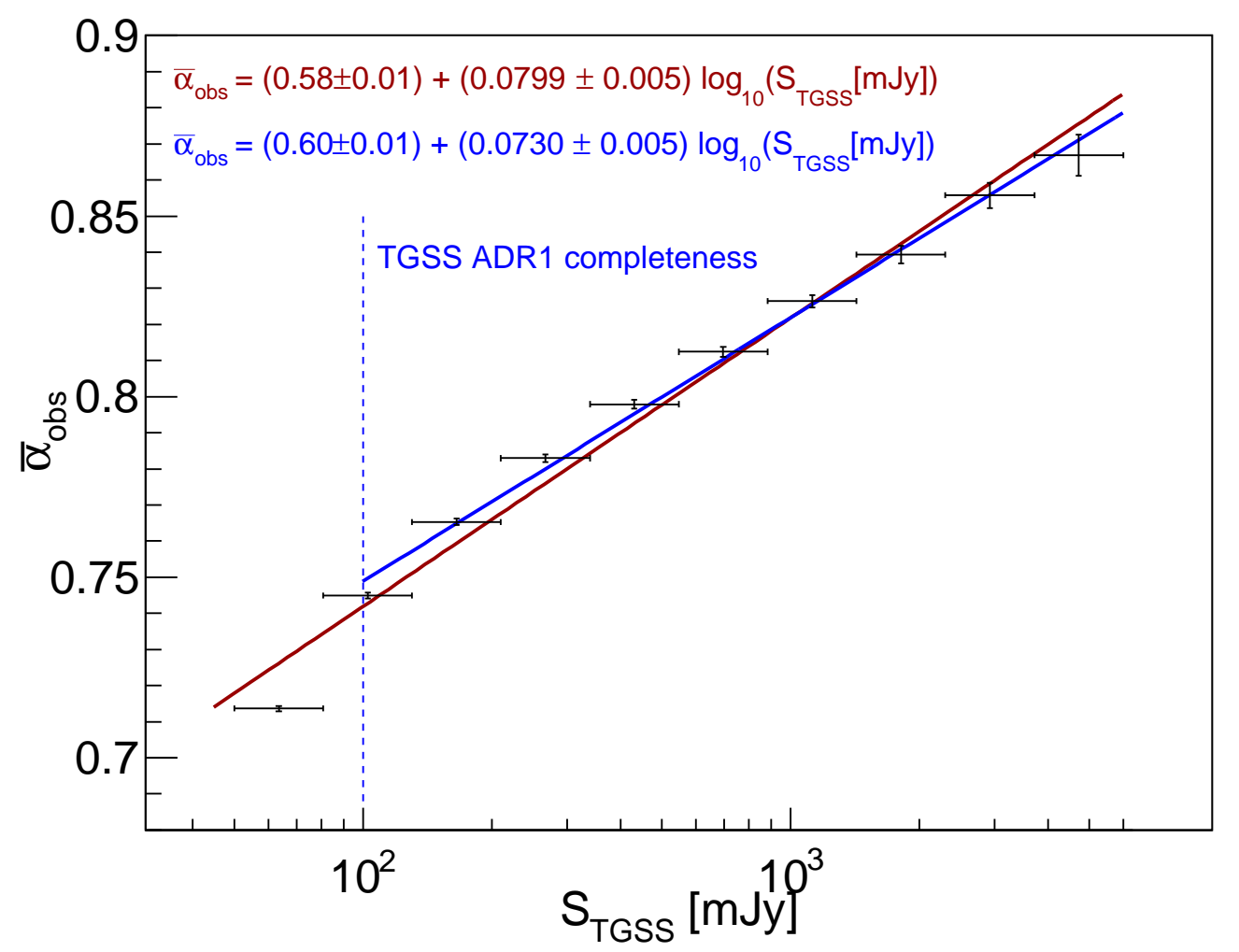

Figure 4: The mean radio spectral index, $\bar{\alpha}_{\text {obs }}$, as a function of the source TGSS flux density. The vertical error-bars are the $1 \sigma$ error in $\bar{\alpha}_{\text {obs }}$ in each flux density bin. The horizontal error-bars represent the width of each bin. The red and blue line represent the fits for full TGSS-NVSS common catalogue and for the common catalogue with TGSS ADR1 flux density completeness, respectively. Fit goodness: $\chi^{2} / \mathrm{NDF}$ is $0.9649 / 7$ (blue) and 5.086/8 (red). Corresponding p-values are 0.9954 (blue) and 0.7484 (red).

Fig. 7 shows $\alpha_{\mathrm{obs}}$ versus $\sqrt{a b}$ for the HF sample. The spectral index increases very rapidly with source size and saturates to the limiting value $\alpha_{\text {obs }}=0.763 \pm 0.211$ at large sizes ( $~\left(35^{\prime \prime}\right.$ ). For ultra large sources, the emission is lobe dominated and therefore $\alpha_{\mathrm{obs}}$ represents the spectral index from lobes. Finally, I plot the $\alpha_{\mathrm{obs}}$ size dependence for sources above and equal $\mathrm{S}_{\mathrm{TGSS}}=200 \mathrm{mJy}$ and $\mathrm{S}_{\mathrm{NVSS}}=40$ mJy in Fig. 8. The spectral index saturates to the limiting value $\alpha_{\text {obs }}=0.763 \pm 0.211$ above sizes $\gtrsim 35^{\prime \prime}$. For these high flux density limits we can be very certain about TGSS completness.

\section{SUMMARY AND DISCUSSION}

I have computed the radio spectral index from a catalogue of about half a million common sources in NVSS and TGSS. The flux density accuracy in the common catalogue is better than $10 \%$ which is sufficient for the studies performed in this paper. I check the robustness of the results with the Galactic plane cuts and find no significant change. I also mask the known peaked-spectrum sources identified from GLEAM survey covering $\sim 60 \%$ of the common catalogue and find no significant change in the results. We have relatively small number of sources close to survey resolution i.e. with size $<25 \operatorname{arcsec}$ ( $\sim 5 \%$ and $1 \%$ of LF and HF sample, respectively) and some fraction of these small size sources may belong to Compact Steep Spectrum (CSS) and Gigahertz Peaked Spectrum (GPS) population (O'Dea 1998; Snellen et al. 2000). In particular, we observe the very flat spectral index $(<0.5)$ for the very 


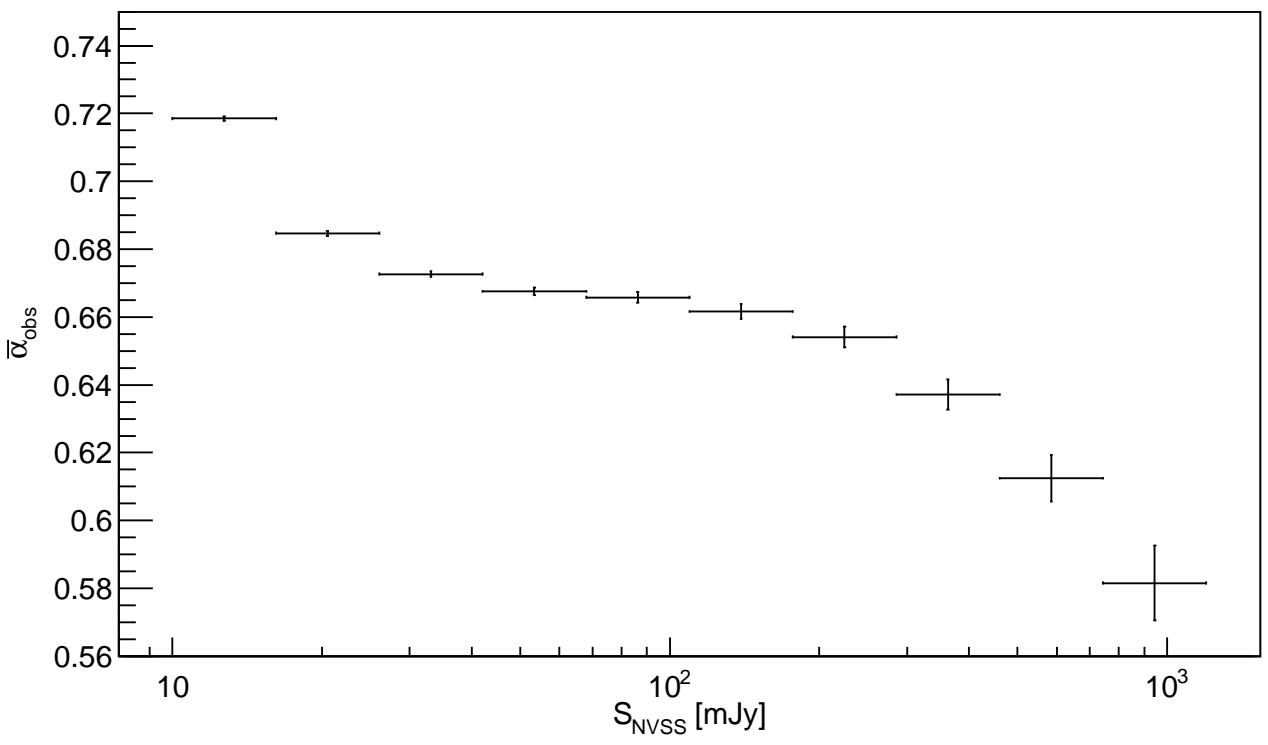

Figure 5: The mean radio spectral index, $\bar{\alpha}_{\text {obs }}$, as a function of the source NVSS flux density. The error bars are as in Fig. 4.

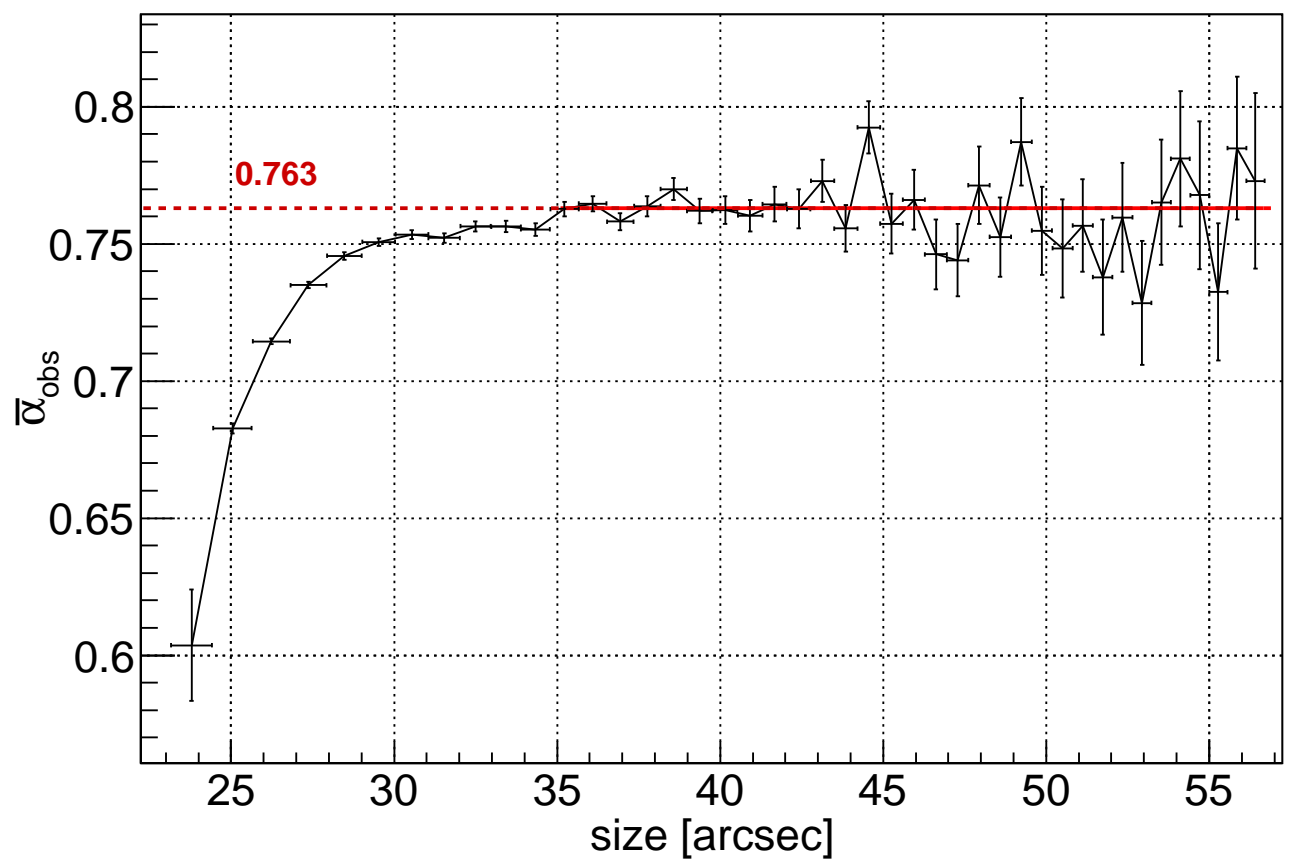

Figure 7: The same as the previous figure but for the HF sources. The red dashed line shows the mean $\alpha_{\text {obs }}$ from sources above 35 arcsec. The $\alpha_{\text {obs }}$ above 35 arcesec for HF sample is $0.763 \pm 0.211$. 


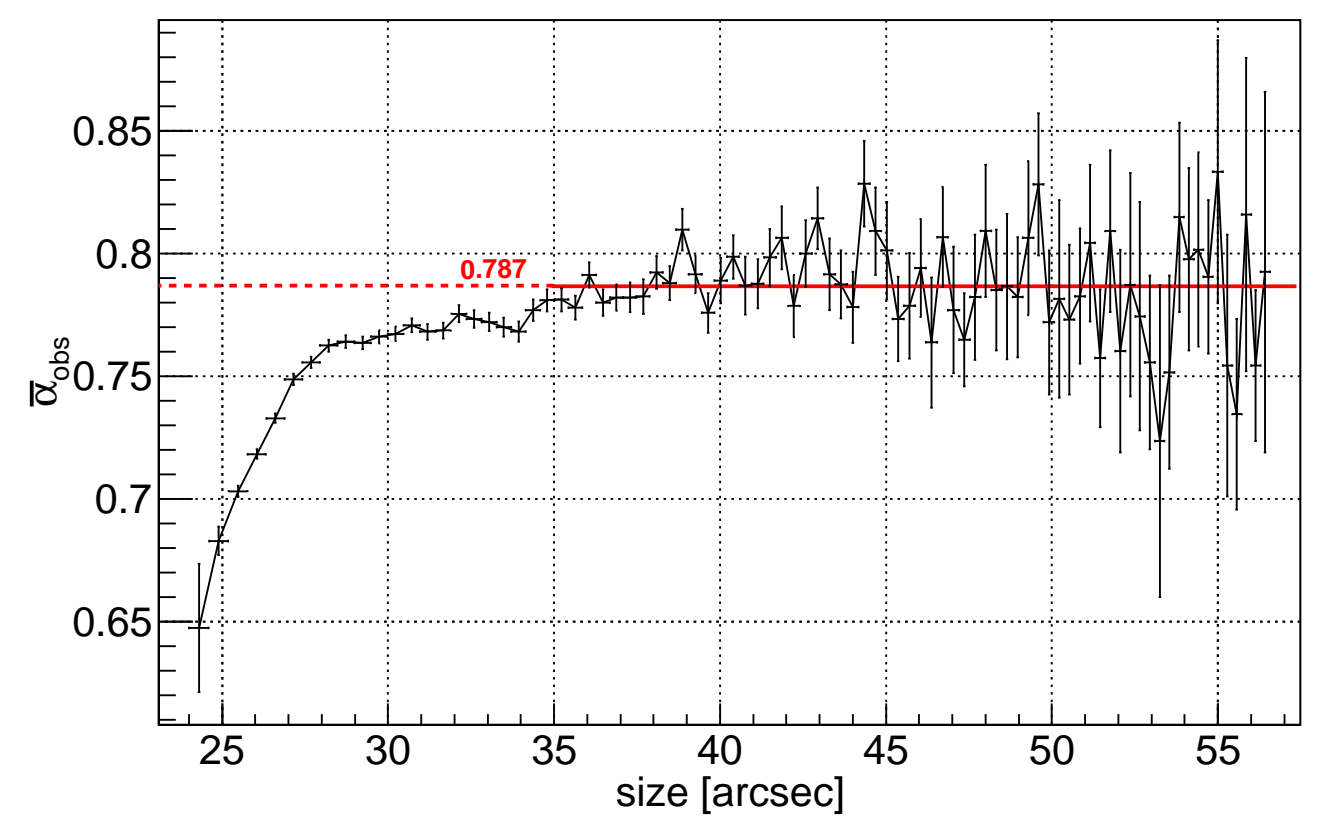

Figure 8: The same as the Fig. 6 but for sources with TGSS flux density $>200 \mathrm{mJy}$.The red dashed line shows the mean $\alpha_{\text {obs }}$ from sources above 35 arcsec. The $\alpha_{\text {obs }}$ above 35 arcesec is $0.787 \pm 0.205$.

compact sources in LF sample, this is likely to be the result of significant CSS or GPS population (Mahony et al. 2016,b). Also, with extreme sizes (small or very large) the orientation is less likely to be randomly oriented. This all together may introduce some bias in the observed spectral index and the results may be relatively less reliable for sources with extreme sizes. The saturated spectral index for both LF and HF sample is robust and independent of these possible systematics and biases though.

I divide the catalogue in two flux density limited samples LF and HF. The large number of sources enables us to study the spectral index in differential size bins. The spatially integrated radio spectral index, relevant to this work, consists of radio emission from lobes and jets. I explore the source size dependence of observed spectral index and draw the following conclusions:

- I confirm the steepening of the radio spectral index in the low frequency bins with increasing flux density (Gopal-Krishna \& Steppe 1982; Steppe \& Gopal-Krishna 1984; Windhorst et al. 1993; Prandoni et al. 2006; Ibar et al. 2009; Ishwara-Chandra et al. 2010; Randall et al. 2012). This trend is inverted if we sort the sources in high frequency flux density bins.

- The spectral index becomes nearly independent of source size above $\sim 44$ arcsec for LF sample and above $\sim 35$ arcsec for HF sample. The saturated spectral index for both LF and HF sample is in between $0.75-0.90$, consistent with previous studies (Intema et al. 2011; Williams et al. 2013, 2016; Hardcastle et al. 2016; Mahony et al. 2016b). Both samples are hosts of AGNs, the LF sample is presumably dominated by FR I galaxies (Wilman et al. 2008). Assuming that the sources lie at redshifts $z \approx 0.8,44$ arcsec size corresponds to $\sim 340 \mathrm{kpc}$ (assuming standard $\Lambda \mathrm{CDM}$ cosmology and using cosmological parameters from Planck Collaboration et al. (2016)). For very compact sources in LF sample, the observed spectral index is flatter $\sim 0.4$, which hints of a significant thermal component from the nucleus in total radio emission for these compact sources. Alternatively, a significant fraction of these compact sources may belong to CSS and GPS population. The CSS and GPS sources may exhibiting a very flat $150 \mathrm{MHz}-1.4 \mathrm{GHz}$ spectral index (Mahony et al. 2016,b). 
The LF sample is increasingly incomplete for low flux densities, even so the results qualitatively represent the low flux density radio sources.

- HF sample exhibits a very strong dependence of the spectral index on size below $\sim 35$ arcsec. This is likely due to an increasing fraction of jets and nuclear radio emission in comparison to emission from lobes. The sources with larger sizes reach a saturated spectral index $\alpha_{\mathrm{obs}}=0.763 \pm 0.211$. The radio emission from these large sources is lobe dominated and so the observed spectral index can be seen as characteristic of radio emission from lobes.

- The saturated spectral index for LF sample is steeper than HF sample. The LF sample is predominantly FR I (Wilman et al. 2008) and the lobes in these galaxies exhibit steepest spectra (Kembhavi \& Narlikar 1999), the electrons in lobes are most retarded and aged. In comparison with HF sample, we see a relatively slow rise of spectral index with size for LF sample. However, I remind the reader that the LF sample is increasingly incomplete for low flux densities and this slow rise of spectral index may be the result of incompleteness. The sharp saturation of spectral index for HF sample, which contains almost equal number of FR I and FR II galaxies (Wilman et al. 2008), indicates very clear domination of radio emission from lobes for these bright galaxies.

\section{ACKNOWLEDGMENTS}

I thank Adi Nusser and Ari Laor for discussion and their extensive and thoughtful comments on this work, and the anonymous referee for useful comments that helped to improve the paper. I especially thank Huib T. Intema for help with the TGSS ADR1 catalogue. This work is supported by NAOC youth talent fund 110000JJ01. I have used CERN ROOT 5.34/21 (Brun et al. 2001) for generating plots.

\section{References}

Antonucci, R. 1993, ARAA, 31, 473

Bell, A. R. 1978, MNRAS, 182, 147

Bell, M. E., Murphy, T., Hancock, P. J., et al. 2019, MNRAS, 482, 2484

Biermann, P. 1976, A\&A, 53, 295

Blandford, R. D., \& Königl, A. 1979, ApJ, 232, 34

Blandford, R. D., \& Ostriker, J. P. 1978, ApJL, 221, L29

Bridle, A. H., \& Perley, R. A. 1984, ARAA, 22, 319

Brun, R., Rademakers, F., et al. 2001, ROOT web page, http: // root.cern.ch/

Callingham, J. R., Ekers, R. D., Gaensler, B. M., et al. 2017, ApJ, 836, 174

Condon, J. J. 1992, ARAA, 30, 575

Condon, J. J., Cotton, W. D., Greisen, E. W., et al. 1998, AJ, 115, 1693

Condon, J. J., \& Yin, Q. F. 1990, ApJ, 357, 97

De Young, D. S. 1976, ARAA, 14, 447

Fan, J.-H., Yang, J.-H., Tao, J., Huang, Y., \& Liu, Y. 2010, PASJ, 62, 211

Fan, J. H., Liu, Y., Yuan, Y. H., et al. 2007, A\&A, 462, 547

Fanaroff, B. L., \& Riley, J. M. 1974, MNRAS, 167, 31P

Gopal-Krishna, \& Steppe, H. 1982, A\&A, 113, 150

Hardcastle, M. J., Gürkan, G., van Weeren, R. J., et al. 2016, MNRAS, 462, 1910

Harwood, J. J., Hardcastle, M. J., \& Croston, J. H. 2015, MNRAS, 454, 3403

Harwood, J. J., Hardcastle, M. J., Croston, J. H., \& Goodger, J. L. 2013, MNRAS, 435, 3353

Harwood, J. J., Croston, J. H., Intema, H. T., et al. 2016, MNRAS, 458, 4443

Harwood, J. J., Hardcastle, M. J., Morganti, R., et al. 2017b, MNRAS, 469, 639

Hurley-Walker, N., Callingham, J. R., Hancock, P. J., et al. 2017, MNRAS, 464, 1146

Ibar, E., Ivison, R. J., Biggs, A. D., et al. 2009, MNRAS, 397, 281

Intema, H. T., Jagannathan, P., Mooley, K. P., \& Frail, D. A. 2017, A\&A, 598, A78

Intema, H. T., van Weeren, R. J., Röttgering, H. J. A., \& Lal, D. V. 2011, A\&A, 535, A38

Ishwara-Chandra, C. H., Sirothia, S. K., Wadadekar, Y., Pal, S., \& Windhorst, R. 2010, MNRAS, 405, 436 
Kellermann, K. I. 1966, ApJ, 146, 621

Kembhavi, A. K., \& Narlikar, J. V. 1999, Quasars and Active Galactic Nuclei An Introduction (Cambridge University Press)

Kirk, J. G., Guthmann, A. W., Gallant, Y. A., \& Achterberg, A. 2000, ApJ, 542, 235

Laing, R. A., \& Bridle, A. H. 2013, MNRAS, 432, 1114

Laing, R. A., \& Bridle, A. H. 2014, MNRAS, 437, 3405

Lal, D. V. 2013, GMRT Observer's Manual, Available at http://gmrt.ncra.tifr.res. in/gmrt_hpage/Users/doc/manual/Manual_2013/manual_20sep2013.pdf

(November 18, 2013)

Lemoine, M., \& Pelletier, G. 2003, ApJL, 589, L73

Mahony, E. K., Morganti, R., Prandoni, I., van Bemmel, I., \& LOFAR Surveys Key Science Project. 2016, Astronomische Nachrichten, 337, 135

Mahony, E. K., Morganti, R., Prandoni, I., et al. 2016b, MNRAS, 463, 2997

Nusser, A., \& Tiwari, P. 2015, ApJ, 812, 85

O’Dea, C. P. 1998, PASP, 110, 493

Ofek, E. O., \& Frail, D. A. 2011, ApJ, 737, 45

Oort, M. J. A., \& Windhorst, R. A. 1985, A\&A, 145, 405

Peterson, B. M. 1997, An Introduction to Active Galactic Nuclei (Cambridge, New York Cambridge University Press)

Planck Collaboration, Ade, P. A. R., Aghanim, N., et al. 2016, A\&A, 594, A13

Prandoni, I., Parma, P., Wieringa, M. H., et al. 2006, A\&A, 457, 517

Randall, K. E., Hopkins, A. M., Norris, R. P., et al. 2012, MNRAS, 421, 1644

Snellen, I. A. G., Schilizzi, R. T., Miley, G. K., et al. 2000, MNRAS, 319, 445

Steppe, H., \& Gopal-Krishna. 1984, A\&A, 135, 39

Swarup, G. 1991, in Astronomical Society of the Pacific Conference Series, Vol. 19, IAU Colloq. 131: Radio Interferometry. Theory, Techniques, and Applications, ed. T. J. Cornwell \& R. A. Perley, 376

Tiwari, P., \& Nusser, A. 2016, Journal of Cosmology and Astroparticle Physics, 2016, 062

Urry, C. M., \& Padovani, P. 1995, PASP, 107, 803

White, R. L., Becker, R. H., \& Helfand, D. J. 2005, AJ, 130, 586

Williams, W. L., Intema, H. T., \& Röttgering, H. J. A. 2013, A\&A, 549, A55

Williams, W. L., van Weeren, R. J., Röttgering, H. J. A., et al. 2016, MNRAS, 460, 2385

Wilman, R. J., Miller, L., Jarvis, M. J., et al. 2008, MNRAS, 388, 1335

Windhorst, R. A., Fomalont, E. B., Partridge, R. B., \& Lowenthal, J. D. 1993, ApJ, 405, 498 